The effectiveness of school-based self-management interventions for asthma among children and adolescents: Findings from a Cochrane systematic review and meta-analysis

Dylan Kneale ${ }^{1}$, Katherine Harris ${ }^{2}$, Vanessa M McDonald ${ }^{3}$, James Thomas ${ }^{1}$, Jonathan Grigg ${ }^{2}$

1. Evidence for Policy and Practice Information and Coordinating Centre (EPPI-Centre), Social Science Research Unit, UCL Institute of Education, University College London, London, UK

2. Centre for Child Health, the Blizard Institute, Queen Mary University of London, UK

3. Centre of Excellence in Severe Asthma, Priority Research Centre for Asthma and Respiratory Disease, School of Nursing and Midwifery, The University of Newcastle, Newcastle, Australia

Corresponding author. Dr Dylan Kneale (D.Kneale@ucl.ac.uk)

Postal Address: Evidence for Policy and Practice Information and Coordinating Centre (EPPICentre), Social Science Research Unit, UCL Institute of Education, 20 Bedford Way, London, UK

Tel: +44 (0)20 76126020

Fax: +44 (0)20 76126020

Keywords: Paediatric Asthma; Self-management; Schools; Systematic Review; Meta-analysis Word count: 3425 


\section{The effectiveness of school-based self-management interventions for asthma among children and adolescents: Findings from a Cochrane systematic review and meta-analysis}

\section{Abstract}

Introduction: The evidence that teaching self-management techniques to children and young people with asthma in schools is effective has not, to date, been the subject of systematic review. Methods: We conducted a systematic review of intervention studies. Studies were eligible if they employed a randomised parallel-group design, and were published in English from 1995 onwards. Participants included 5-18 year old children with asthma who participated within their own school environment. Searches were conducted on the Cochrane Airways Group Specialised Register. Quantitative data were combined using random-effects meta-analyses.

Results: Thirty-three outcome evaluation studies were included. School based interventions were effective in reducing the frequency of emergency department visits (OR 0.70, 95\% CI 0.53 to 0.92 ; studies $=13$ ), and moderately effective in reducing levels of hospitalisations (SMD -0.19, $95 \%$ CI -0.35 to -0.04 ; studies=6). A meta-analysis of three studies suggest that the intervention approach could reduce the number of days of restricted activity (SMD - $0.30,95 \%$ CI - 0.41 to 0.18; studies=3). However, there was uncertainty as to whether school-based self-management interventions impacted on reducing absences from school.

Conclusions: Self-management interventions for children with asthma delivered in schools reduce the number of acute episodes of healthcare usage. We conclude that the school environment is an important space for delivering interventions to improve children's health. 
- Do schools provide an effective space for teaching children with asthma the selfmanagement techniques they need in order to lower their levels of healthcare usage, improve their asthma symptomology, and lower their levels of school absences?

What is the bottom line?

- Meta-analysis of randomised controlled trial data shows that children who receive school-based asthma self-management interventions have reduced levels of hospitalisations, emergency department visits, and show an improvement in days of restricted activity. However, no impact was observed on levels of school absence.

\section{Why read on?}

- This is the first systematic review and meta-analysis of school-based asthma interventions. 


\section{Introduction}

Asthma is the most common chronic non-communicable disease among children ${ }^{1}$ and, in England, almost one in six children aged 5-14 years will have experienced asthma at some point ${ }^{2}$, with substantial impacts on their health-related quality of life. For example over 2.8 million school days per annum are estimated to be lost in the UK due to asthma and asthmatic symptoms ${ }^{3}$. Well-controlled asthma consists of reduced daytime and night-time symptoms, decreased long-term morbidity and diminished risk of life-threatening asthma attacks ${ }^{4}$. Selfmanagement, a cornerstone of treatment for people with asthma ${ }^{5}$, is the systematic process of educating and enabling individuals to achieve good control of their asthma symptoms, thereby preventing future exacerbations ${ }^{6}$.

Schools are postulated to be effective sites for teaching asthma self-management techniques to children due to their familiarity as environments for learning, and the potential for identification of large numbers of children with asthma at a single school site ${ }^{7-9}$. However, few reviews exist that have focused explicitly on schools as delivery sites, or have synthesised information across studies using meta-analysis. There consequently remains uncertainty as to the impact of providing self-management education and support within schools. In this systematic review, we therefore aimed to identify and synthesise evidence on school-based self-management interventions for children with asthma, with a focus on effectiveness. Although delivery of an asthma self-management intervention in schools is a potential way of reducing asthma burden in children and young people, to date uncertainty remains as to the effectiveness of this approach across a number of different outcomes $\left({ }^{1011}\right)$. 


\section{Methods}

Full details of the methods used were published on registration of the protocol for this review through the Cochrane Collaboration (CD011651) ${ }^{12}$. The complete review aimed to address two key objectives: (i) identify the key design features and processes associated with successful implementation of school-based asthma self-management interventions; (ii) understand whether school-based interventions can effectively change asthma self-management behaviour. In this manuscript, we focus on the quantitative synthesis of evidence on the effectiveness school-based asthma interventions. Complete details, including the results of the review of intervention design and implementation factors can be found in the full companion Cochrane review, which is published simultaneously with this paper ${ }^{11}$.

\section{Study eligibility}

Studies were eligible if they employed a randomised parallel-group design with children randomised individually or in clusters, and were published in English from 1995 onwards (this date corresponding with publication of the first Global Initiative for Asthma (GINA) guidelines, which provided a foundation for asthma guidelines globally ${ }^{13}$. Participants included school-aged (five-year-old to 18-year-old) children and young people with asthma who participated within their own school environment.

All included interventions aimed to develop and enhance self-management of asthma among children through at least one of the following components: (i) increasing knowledge of asthma 
and its management; (ii) enhancing self-management skills; (iii) improving self-management behaviours and practice. The definition of self-management used followed British Thoracic Society (BTS) guidelines ${ }^{14}$ and eligible studies included the development of at least one of the following components: reinforcement of regular lung function monitoring; development of an therapeutic alliance between patients and healthcare practitioners; instruction on inhaler techniques; reinforcement/provision of an individualised written asthma management plan; instruction on the appropriate use of reliever and preventer therapies; and selected nonpharmacological strategies. Thus, an intervention where school nurses directly delivered medication to children or directly observed therapy (e.g. Halterman et al ${ }^{15}$ ), but which did not include a component aimed at developing self-management skills, would be excluded (see supplementary materials). Self-management interventions could either be provided by a trained educator, or nurse (including school, practice or community nurse), or doctor or physician, or peer or social worker, (or a combination of these). Eligible studies also included a comparison group that either received usual care or a self-management or health intervention with a focus other than asthma (placebo).

\section{Selecting and combining outcomes}

Information on four primary outcomes, reflective of indicators of good asthma control ${ }^{14}$, were extracted: (i) exacerbations or asthma attacks leading to admission to hospital (hospitalization); (ii) asthma symptoms leading to emergency hospital visits; (iii) absence from school; (iv) days of restricted activity. Information on a number of secondary outcomes was also extracted and synthesized ${ }^{11}$. 
A search was conducted on the Cochrane Airways Group Specialised Register, which covers a number of databases including CENTRAL, MEDLINE, EMBASE, PsycInfo and CINAHL as well as respiratory conference abstracts, for trials, using a strategy outlined in the full review ${ }^{11}$ and developed by the Airways Group Trials Search Co-ordinator (details in acknowledgements). All results were imported into EPPI-Reviewer 4 (systematic review software ${ }^{16}$ ) and screened on title and abstract by two reviewers (DK and $\mathrm{KH}$ ). Full texts were obtained for studies meeting initial eligibility criteria and rescreened by both reviewers, who met to discuss and consolidate any disagreements. All studies meeting the criteria for study design were included, irrespective of the outcomes that were collected. All data were extracted into EPPI-Reviewer 4, which was also used for calculating effect sizes including adjustments for clustering ${ }^{16}$; STATA was used for conducting further data transformations and robustness checks ${ }^{17}$; while RevMan 5.3 was the primary software used for combining quantitative data ${ }^{18}$. All data were initially combined using random-effects meta-analyses, as the underlying assumptions of a fixed-effects specification were not deemed to be compatible with the likely heterogeneity in intervention types and populations across studies.

Adjustments for clustering were made where this was not reported by trialists, and because no study included in the meta-analysis provided a direct estimate of the clustering effect through an intracluster correlation coefficient (ICC), an estimate of 0.05 was selected based on the ICC used in a study not included in the meta-analysis ${ }^{19}$. We expected outcomes to be reported using similar units of analysis, although in reality we encountered a number of variations and used 
Chinn's formula ${ }^{20}$ for converting effect sizes and standard errors between standardized mean differences and odds ratios, following direction provided in the Cochrane Handbook ${ }^{21}$.

We assessed statistical heterogeneity through examining the $\mathrm{I}^{2}$ measure and Cochran's $\mathrm{Q}^{21}$, and explored drivers of heterogeneity through conducting pre-specified sensitivity and subgroup analyses. We intended to construct random-effects meta-regression models, which would allow us to explore the impact of different covariates simultaneously. However, a relatively low number of studies (our largest model included 13 studies) meant that we were unable to extend the modelling in this way. We assessed sources of potential bias using the Cochrane Collaboration's Risk of Bias tool ${ }^{22}$. For publication bias, we plotted the distribution of studies' effect sizes against their standard errors in a funnel plot for each outcome; we also undertook formal tests for small-study publication bias using Egger's test ${ }^{23}$. However, these tests were likely underpowered for at least two of the outcomes (hospitalisations and days of restricted activity). Further sensitivity analyses were undertaken on the basis of the study's risk of bias assessment, the impact of using fixed-effects compared to random effects modelling, and the impact of studies using a cluster randomised design.

\section{Results}

The first search was conducted in April 2015, with updated searches conducted in April 2016 and 2017. After de-duplication, the titles and abstracts of 379 outcome evaluation studies were independently screened by two review authors (KH and DK). Following exclusion on title and 
abstract, the remaining 105 full-text records were assessed for eligibility, and thirty-three outcome evaluation studies were included for further analysis (figure 1).

\section{FIGURE 1 - FLOW CHART HERE}

The majority of studies (79\%) randomised children by school; in contrast just seven studies randomised by individuals. The evidence was mainly informed by studies that had taken place in North America (24 in the USA and 4 in Canada). Of the remaining, two studies had taken place in the UK, two in Australia and one study each in China, Spain and Jordan. Eight studies took place in high/senior/secondary schools, three in junior/middle schools, and fifteen in primary/elementary schools; a further three studies had taken place in a mixture of schools with the remainder being unclear (table 1). School-based asthma interventions took a number of diverse forms - from more manualised programmes including the American Lung Association's Open Airways for Schools programme (9 studies), Roaring Adventures of Puff (4 studies), and the Triple-A programme (Adolescent Asthma Action; 2 studies) - to other novel intervention models being trialled for the first time. A minority of interventions were of relatively low intensity, comprising just one session ( 2 studies) although most were of higher intensity comprising three or more sessions (28 studies, with three providing insufficient description). In nine studies the interventions were delivered fully or in part by a school or public health nurse or nursing student, four involved teachers in delivery, and two studies involved peer delivery (see supplementary materials). 
Study quality varied according to domain, and for some domains, the risk of bias was deemed to be high in studies (see the full review for further details ${ }^{11}$ ). However, the overall the potential for this risk of bias to compromise the results of the meta-analyses for the primary outcomes was deemed to be 'not serious' when grading the quality of the evidence and the strength of the recommendations.

\section{TABLE 1 HERE - RESULTS}

Enough information on exacerbations leading to hospital to calculate an effect size was provided by seven studies, six of which were combined in a meta-analysis of standardised mean differences (SMD), with all six studies reporting a positive impact of the intervention and little evidence of statistical heterogeneity based on $\mathrm{I}^{2}(0 \%)$ and Cochran's Q statistics (table 1 and figure 2). Overall, the results suggested that school-based asthma self-management interventions were moderately effective in reducing levels of hospitalisations among children (SMD -0.19, $95 \%$ CI -0.35 to -0.04$)$. Tests for publication bias were ultimately underpowered, although no evidence of publication bias was detected through visual inspection of the data.

A further measure of healthcare usage, emergency department (ED) visits was also included as a primary outcome, with data transformed to reflect the differential odds of reports of ED visits between intervention and control groups. Based on the results from 13 studies involving 3883 children, the evidence suggested that school-based asthma self-management interventions were effective in reducing the frequency of emergency department visits (ED visits; (OR 0.70, 95\% CI 0.53 to 0.92 ); figure 3 ). Among the thirteen included studies, there was substantial heterogeneity 
however, both in terms of magnitude and direction of effect, with three studies having negligible effect sizes (close to zero) and two having negative effect sizes; this resulted in an $\mathrm{I}^{2}$ of $26 \%$. The number of studies and the level of heterogeneity allowed us to explore potential study characteristics that could help to explain the observed variation through planned sub-group analyses, although children's age, school type and socioeconomic status of children did not explain the observed heterogeneity. Sensitivity analyses based on model assumptions and data transformations did not suggest that the analytical decisions made influenced the effect sizes, and although based on a relatively small number of studies, neither the funnel plot nor Egger's test were indicative of publication bias.

\section{FIGURE 2 HERE}

FIGURE 3 HERE

Included in our meta-analysis of effects on school absences were 10 studies, although there was uncertainty as to whether school-based self-management interventions impacted on reducing absences from school (SMD -0.07, 95\% CI -0.22 to 0.08). Among the studies, there was very high heterogeneity between effect size estimates, with $\mathrm{I}^{2}$ estimated at $70 \%$. Effect sizes from half of the studies included in the meta-analysis indicated that the intervention had a negative impact in slightly or significantly increasing the number of school absences in the intervention group relative to the control. Pre-specified sub-group analyses generally did not suggest that studylevel characteristics explained between study heterogeneity, although one sub-group analysis (described in the full Cochrane review ${ }^{11}$ ) indicated that studies drawing upon a defined theoretical framework were more effective (SMD $-0.20,95 \%$ CI -0.36 to -0.04 ; studies $=6$ ) than 
those that did not (SMD 0.08, 95\% CI -0.05 to 0.20; studies =4); although there remained moderate levels of heterogeneity for both sub-groups (figure 4). Sensitivity analyses based on model assumptions and data transformations did not suggest that the analytical decisions made influenced the effect sizes, and there was negligible evidence of publication bias.

\section{FIGURE 4}

\section{FIGURE 5}

Finally, three studies contributed data to our meta-analysis of the impact of school-based asthma self-management interventions in reducing the number of days of restricted activity that children experienced (Figure 5). These provided evidence that the intervention mode could reduce the number of days of restricted activity experienced (SMD $-0.30,95 \%$ CI -0.41 to -0.18 ; studies $=$ 3), albeit based on a limited number of studies. All three studies provided relatively consistent evidence around the direction and magnitude of the effect (I $2=0 \%)$.

\section{Discussion}

The results from the meta-analyses showed that school-based self-management interventions are effective in improving healthcare usage including hospitalisations (6 studies) and ED visits (13 studies) as well as effective in improving an indicator of health status in reducing days of restricted activity (3 studies). Effectiveness was not consistent for school absences, where the point estimate suggested a small effect and with a confidence interval that crossed the line of no effect. 
The intervention appeared most effective for outcomes involving healthcare usage. These were measured in a relatively uniform way in studies (although several transformations were made in the meta-analyses to facilitate synthesis). The reason why a smaller impact was observed for school absences is unclear, although additional subgroup analyses suggested that the way in which the intervention was implemented may have a greater impact on this outcome. In particular, the reported use of a theoretical framework in the study appeared to distinguish between studies that were more effective in reducing levels of school absences compared to those that were not. While there is no universal consensus as to the importance of school absences as an indicator of asthma control ${ }^{24}$, demonstrating improvement on this indicator may be important in gaining access to school sites and the cooperation of school staff. This result does not indicate that use of theory is causal in reducing school absences; however, potential explanations might include that where trialists have an in-depth knowledge of how their intervention works, which shapes all stages of delivery through to the reporting of the outcomes, that this leads to better outcomes for children, particularly for outcomes that may be otherwise more intractable; alternatively the reported use of theory may be a marker for the experience of the trialists, or trial team.

There are several limitations to this review. First, most of the studies were conducted in the USA and Canada, and very few of the included studies were conducted in the UK, Europe, or beyond. Factors reflecting health policy and access to healthcare across different settings are likely to influence the design and implementation of an intervention, although their impact was not fully assessed here. Nevertheless, the way in which children attend schools is fairly similar worldwide, therefore little impact is expected in terms of the applicability of the approach to schools in middle and higher income settings. However, the US-focus of studies may impact on the 
transferability of the intervention findings. The nature of healthcare delivery, and the high number of children without adequate healthcare coverage could mean that the intervention has a greater impact in US settings compared to settings with universal healthcare coverage (e.g. the UK), particularly among lower income populations with substantial levels of under-diagnosis and low levels of access to the correct medication plans. Several of the trials (e.g. ${ }^{25} 26$ were developed precisely on the basis of this rationale, and selected schools as the delivery site because of the universality of education, as opposed to healthcare, in these settings. The implications for transferability could mean that lower effect sizes are achieved in settings with better healthcare coverage, higher rates of diagnosis, and greater equality in access to appropriate medication (e.g. settings such as the UK where healthcare is universally free at the point of delivery). Similarly, a greater number of studies were conducted within primary/elementary schools than within high/secondary schools. Both the underrepresentation of settings beyond North America and high school settings represent caveats to the generalisability of findings.

A second limitation is that there was variation in the way in which a number of outcomes were measured. Previous calls to action have been made to standardise the outcome that are collected during children's asthma trials ${ }^{27-29}$, although this review suggests that these have only been partially successful, given that data from a number of studies that met the eligibility criteria for trial design were not included in meta-analysis because the trials did not measure these outcomes. While the outcomes included in the meta-analyses were only combined after a lengthy consideration of the potential methodological and clinical heterogeneity, there also remains a likelihood that at least part of the heterogeneity observed between studies was due to measurement error. For many of the secondary outcomes that were examined in the companion review $^{11}$, for example lung function, there was even greater heterogeneity in the measurement of 
outcomes that precluded combining the effect sizes within a meta-analytic framework. In addition to greater efforts for standardisation of outcome domains, this review suggests a need for greater standardisation of appropriate measurement and reporting of outcomes collected within trials. For example, in the case of hospitalisations, the original data were collected in studies as the mean number of hospitalisations (continuous count data), reports of any instances of hospitalisations (binary data), and median level of hospitalisation (the latter not combined in meta-analysis); these also differed in measurement approach (hospitalisations over a relatively short duration (e.g. 4 weeks $^{30}$ ) compared to a longer exposure period (e.g. 12 months ${ }^{31}$ )). Agreement on which outcomes to measure may represent an important first step in standardising trial reporting and developing core outcome sets ${ }^{32}$, however this review suggests that further work is needed to agree how these indicators should be operationalised. The diversity of measurement approaches, and consequently the number of transformations needed to standardise the data into a common metric, do potentially compromise the results. While sensitivity analyses were conducted in full to check the robustness of our assumptions, the lack of standardisation nevertheless represents a caveat to the results.

A third limitation is that, since we excluded studies that delivered other asthma interventions, we do not know the added value of running an intervention in a school, compared with running an intervention in a hospital or community setting. Similarly, we excluded studies that delivered asthma interventions in schools, but that did not meet our definition of self-management. This may have narrowed our ability to understand the full potential of schools as sites for improving children's asthma. What is clear, however, is that schools provide access to large numbers of children with asthma, including those who do not regularly attend appointments with their medical provider. 
A fourth limitation is that, while the overall impact of school-based interventions was positive for most of the primary outcomes examined in the review, some individual studies did report negative intervention impacts among children. For example, in our model of ED visits, two studies, both using the same intervention model, reported that children were more likely to experience an ED visit after the intervention than the control. The underlying mechanisms of how a school-based asthma intervention increases healthcare usage are unclear. Such potentially counter-intuitive effects may reflect the content of self-management information delivered to children, which could recommended greater contact with healthcare providers when children experience asthma exacerbations. A narrative approach to the synthesis of the outcome evaluations data including reviewing the content of the self-management education could lead to an enhanced understanding of why a small number of trials ostensibly led to more negative outcomes among children.

In summary, this review supports the hypothesis that school-based self-management interventions are effective in improving healthcare usage outcomes for children with asthma. Although we found no conclusive evidence in the meta-analysis for the impact of the intervention on school absences, results from the subgroup analyses suggest that a subgroup of studies that explicitly defined their theoretical framework had higher effect sizes. Future systematic reviews that can draw on a larger pool of studies may be able to further identify the importance of different configurations on the effectiveness of the intervention, and it may be possible to use Network Meta-Analyses, drawing on the relatively large number of studies for some intervention modes (e.g. Open Airways for Schools), to compare indirectly and directly how different intervention approaches perform. Since self-management interventions delivered in schools reduce the number of acute episodes of healthcare usage in particular, we conclude 
that the school environment is an important space for delivering interventions aimed at improving asthma outcomes in children and young people.

\section{Acknowledgements}

This research was funded by the National Institute for Health Research (NIHR) Collaboration for Leadership in Applied Health Research and Care North Thames at Bart's Health NHS Trust (NIHR CLAHRC North Thames). The views expressed in this article are those of the author(s) and not necessarily those of the NHS, the NIHR, or the Department of Health and Social Care.

Thanks to Elizabeth Stovold for helping to define the search strategy. Thanks also to Emma Dennett and Chris Cates for comments and ongoing assistance. Thanks to the NIHR Collaborative Leadership in Applied Health Research and Care (CLAHRC) North Thames for continued support

\section{Contributorship}

DK, KH, VM, JT, JG contributed to study conception and protocol of the review. DK, KH performed the systematic review. DK and KH performed the statistical analysis. All authors contributed to the interpretation of data. DK and $\mathrm{KH}$ drafted the paper and all authors provided critical revisions and contributed to the editing of the paper. DK and KH are equal contributors to this manuscript.

\section{Funding}

This research was funded by the National Institute for Health Research (NIHR) Collaboration for Leadership in Applied Health Research and Care North Thames at Bart's Health NHS Trust (NIHR CLAHRC North Thames). The views expressed in this article are those of the author(s) 
and not necessarily those of the NHS, the NIHR, or the Department of Health and Social Care. Competing interests

Dr. Kneale reports grants from NIHR CLAHRC North Thames, during the conduct of the study; Ms. Harris has nothing to disclose; Dr. McDonald has nothing to disclose; Dr. Thomas reports grants from NIHR, during the conduct of the study; Dr. Grigg reports personal fees from GSK, personal fees from Vifor Pharmaceuticals, outside the submitted work

\section{Exclusive licence}

The Corresponding Author has the right to grant on behalf of all authors and does grant on behalf of all authors, an exclusive licence (or non-exclusive for government employees) on a worldwide basis to the BMJ Publishing Group Ltd and its Licensees to permit this article (if accepted) to be published in Thorax and any other BMJPGL products to exploit all subsidiary rights, as set out in our licence http://journals.bmj.com/site/authors/editorial-policies.xhtml\#copyright and the Corresponding Author accepts and understands that any supply made under these terms is made by BMJPGL to the Corresponding Author. Where the Corresponding Author wishes to make the article available on an open access basis (and intends to pay the relevant open access fee), the terms of such open access shall be governed by a Creative Commons licence - details of these licences and which Creative Commons licence will apply to this article are set out in our licence referred to above. The Corresponding Author is one author signing on behalf of all co-owners of the Contribution. The Corresponding Author confirms this manuscript has not been accepted for publication elsewhere, is not being considered for publication elsewhere and does not duplicate material already published. The Corresponding Author confirms all authors consent to publication of this manuscript. 


\section{References}

1. Asher I, Pearce N. Global burden of asthma among children. The international journal of tuberculosis and lung disease 2014;18(11):1269-78.

2. Simpson CR, Sheikh A. Trends in the epidemiology of asthma in England: a national study of 333,294 patients. Journal of the Royal Society of Medicine 2010;103(3):98-106.

3. Mukherjee M, Stoddart A, Gupta RP, et al. The epidemiology, healthcare and societal burden and costs of asthma in the UK and its member nations: analyses of standalone and linked national databases. BMC medicine 2016;14(1):113.

4. Juniper EF, Bousquet J, Abetz L, et al. Identifying 'well-controlled' and 'not well-controlled' asthma using the Asthma Control Questionnaire. Respiratory medicine 2006;100(4):616-21.

5. Network BTSSIG. British guideline on the management of asthma. Thorax 2008;63:iv1.

6 . Kotses H, Creer TL. Asthma self-management. Asthma, Health and Society. New York City: Springer 2010:117-39.

7. Ahmad E, Grimes DE. The effects of self-management education for school-age children on asthma morbidity: a systematic review. The Journal of School Nursing 2011:1059840511403003.

8. Bruzzese J-M, Evans D, Kattan M. School-based asthma programs. Journal of Allergy and Clinical Immunology 2009;124(2):195-200.

9. Coffman JM, Cabana MD, Yelin EH. Do school-based asthma education programs improve selfmanagement and health outcomes? Pediatrics 2009;124(2):729-42.

10. Kneale D, Thomas J, Harris K. Developing and Optimising the Use of Logic Models in Systematic Reviews: Exploring Practice and Good Practice in the Use of Programme Theory in Reviews. PloS one 2015 doi: DOI: 10.1371/journal.pone.0142187

11. Harris KM, Kneale D, Lasserson TJ, et al. School-based self management interventions for asthma in children and adolescents: a mixed methods systematic review. The Cochrane Library in press

12. Harris KM, Kneale D, Lasserson TJ, et al. School-based self management interventions for asthma in children and adolescents: a mixed methods systematic review. The Cochrane Library 2015 doi: 10.1002/14651858.CD011651

13. National Heart, Lung, and Blood Institute. Global Initiative for Asthma Management and Prevention NHLBI/WHO Workshop Report; 1995. National Institutes of Health, National Heart Lung, and Blood Institute.

14. BTS BTS. British guideline on the management of asthma: A national clinical guideline. London: British Thoracic Society, 2012.

15. Halterman JS, Szilagyi PG, Fisher SG, et al. Randomized controlled trial to improve care for urban children with asthma results of the school-based asthma therapy trial. Arch Pediatr Adolesc Med 2011;165(3):262-68.

16. Thomas J, Brunton J, Graziosi S. EPPI-Reviewer 4.0: software for research synthesis. London: Institute of Education, 2010.

17. Stata Statistical Software: Release 13 [program]. College Station, TX: StataCorp LP, 2013.

18. Review Manager (RevMan) 5.3 [program], 2008.

19. McCann D, McWhirter J, Coleman H, et al. A controlled trial of a school-based intervention to improve asthma management. European Respiratory Journal 2006;27(5):921-28. 
20. Chinn S. A simple method for converting an odds ratio to effect size for use in meta-analysis. Statistics in medicine 2000;19(22):3127-31.

21. Higgins JPT, Green S. Cochrane handbook for systematic reviews of interventions. Chichester: WileyBlackwell 2011.

22. Higgins JP, Altman DG, Gøtzsche PC, et al. The Cochrane Collaboration's tool for assessing risk of bias in randomised trials. Bmj 2011;343:d5928.

23. Harbord RM, Harris RJ, Sterne JAC. Updated tests for small-study effects in meta-analyses. Stata Journal 2009;9(2):197.

24. Harris K, Mosler G, Williams SA, et al. Asthma control in London secondary school children. Journal of Asthma 2017:1-8.

25. Bruzzese J-M, Sheares BJ, Vincent EJ, et al. Effects of a school-based intervention for urban adolescents with asthma: a controlled trial. American journal of respiratory and critical care medicine 2011;183(8):998-1006.

26. Gerald LB, McClure LA, Mangan JM, et al. Increasing adherence to inhaled steroid therapy among schoolchildren: randomized, controlled trial of school-based supervised asthma therapy. Pediatrics 2009;123(2):466-74.

27. Reddel HK, Taylor DR, Bateman ED, et al. An official American Thoracic Society/European Respiratory Society statement: asthma control and exacerbations: standardizing endpoints for clinical asthma trials and clinical practice. American journal of respiratory and critical care medicine 2009;180(1):59-99.

28. Sinha IP, Gallagher R, Williamson PR, et al. Development of a core outcome set for clinical trials in childhood asthma: a survey of clinicians, parents, and young people. Trials 2012;13(1):103.

29. Centers for Disease Control. National Asthma Control Program: Improving Quality of Life and Reducing Costs. Atlanta, USA: Centers for Disease Control, 2005.

30. Atherly A, Nurmagambetov T, Williams $S$, et al. An economic evaluation of the school-based "Power Breathing" asthma program. Journal of Asthma 2009;46(6):596-99.

31. Horner SD, Fouladi RT. Improvement of Rural Children's Asthma Self-Management by Lay Health Educators. Journal of School Health 2008;78(9):506-13.

32. Williamson PR, Altman DG, Blazeby JM, et al. Developing core outcome sets for clinical trials: issues to consider. Trials 2012;13(1):132. 
Table 1: Summary of meta-analyses

\begin{tabular}{|c|c|c|c|c|c|}
\hline Outcome & \begin{tabular}{|c|} 
Number \\
of \\
Studies
\end{tabular} & \begin{tabular}{|l|} 
Number of \\
Participants
\end{tabular} & $\begin{array}{c}\text { Effect size } \\
(\text { modelling strategy })\end{array}$ & \begin{tabular}{||c|} 
Pooled effect size \\
and Confidence \\
Interval
\end{tabular} & $\overline{\mathbf{I}^{2}}$ \\
\hline $\begin{array}{l}\text { Exacerbations } \\
\text { leading to } \\
\text { hospitalisation }\end{array}$ & 6 & 1873 & $\begin{array}{l}\text { Std. Mean } \\
\text { Difference (IV, } \\
\text { Random effects, } \\
95 \% \text { CI) }\end{array}$ & $\begin{array}{l}-0.19[-0.35,- \\
0.04]\end{array}$ & $0 \%$ \\
\hline $\begin{array}{l}\text { Exacerbations } \\
\text { leading to } \\
\text { emergency } \\
\text { department (ED) } \\
\text { visits }\end{array}$ & 13 & 3883 & $\begin{array}{l}\text { Odds Ratio (IV, } \\
\text { Random effects, } \\
95 \% \text { CI) }\end{array}$ & $0.70[0.53,0.92]$ & $26 \%$ \\
\hline Absence from school & 10 & 4609 & $\begin{array}{l}\text { Std. Mean } \\
\text { Difference (IV, } \\
\text { Random effects, } \\
95 \% \text { CI) }\end{array}$ & $\mid-0.07[-0.22,0.08]$ & $70 \%$ \\
\hline $\begin{array}{l}\text { Days of restricted } \\
\text { activity }\end{array}$ & 3 & 1852 & $\begin{array}{l}\text { Std. Mean } \\
\text { Difference (IV, }\end{array}$ & $\begin{array}{l}-0.30[-0.41,- \\
0.18]\end{array}$ & $0 \%$ \\
\hline
\end{tabular}




\begin{tabular}{||l|l|l|l|l|||}
\hline & & Random effects, & \\
$95 \% \mathrm{CI})$ & & \\
\hline
\end{tabular}

Key: IV: Inverse Variance; CI: Confidence Interval 